\title{
Reforma da Atenção Primária em Portugal em duplo movimento: unidades assistenciais autónomas de saúde familiar e gestão em agrupamentos de Centros de Saúde
}

\author{
Primary Healthcare Reform in Portugal on two fronts: \\ autonomous family healthcare units \\ and management of groupings of Health Centers
}

Luis Pisco ${ }^{1}$

${ }^{1}$ Centro de Saúde da Foz do Arelho. Rua Francisco Almeida Grandela 8Q. 2500-487 Foz do Arelho Portugal.

luispisco@mail.telepac.pt

\begin{abstract}
In 2005, Portugal began a reform of Primary Health Care. This reform process through to April 2010 is described and analyzed. During this period the Mission for Primary Health Care was responsible for conducting a profound reconfiguration. The main objectives for this reform were to improve accessibility, efficien$c y$, quality and continuity of care and increase the satisfaction of professionals and citizens. The main features are voluntary adhesion, teamwork, mandatory information system, performancesensitive payment, contracting and evaluation. The reconfiguration of health centers was two pronged. First, there was the formation of small autonomous functional units, known as Family Health Units (USF) providing services with proximity and quality. The second measure involved the aggregation of resources and management structures, groups of health centers (ACES), seeking to achieve efficiency and economies of scale. The FHU proved to offer simultaneously more efficiency, accessibility, better working environment, greater citizen satisfaction, namely better quality. The importance of strong political support, the creation of a structure responsible for the design and implementation of reform and good liaison with the media are stressed.

Key words Reform of Primary Health Care, Family health units, Health center groupings, Autonomy
\end{abstract}

Resumo Analisa-se o processo de reforma da atenção primária à saúde (APS) em Portugal de 2005 a abril de 2010, período em que a Missão para os Cuidados de Saúde Primários teve a responsabilidade de conduzir essa profunda reconfiguração da APS Portuguesa. Os principais objectivos da reforma foram: melhorar a acessibilidade, eficiência, qualidade e continuidade dos cuidados e aumentar a satisfação dos profissionais e cidadãos. Suas principais características são a adesão voluntária, trabalho em equipa, existência obrigatória de sistema de informação, pagamento por desempenho, contratualização e avaliação. A reconfiguração dos centros de saúde obedeceu a um duplo movimento: por um lado, formação de pequenas unidades funcionais autónomas, as Unidades de Saúde Familiar (USF), prestando serviços com proximidade e qualidade; por outro lado, a agregação de recursos e estruturas de gestão, os agrupamentos de Centros de Saúde (ACES) visando a eficiência e economia de escala. As USF conseguiram simultaneamente mais eficiência, acessibilidade, melhor clima laboral, maior satisfação dos cidadãos, numa palavra, mais qualidade. Salienta-se a importância de um forte apoio político, da criação de uma estrutura responsável pelo desenho e implementação da reforma e de uma boa comunicação social.

Palavras-chave Reforma da Atenção Primária, Unidades de saúde familiar, Agrupamentos de centros de saúde, Autonomia 
The difficulty lays not so much in developing new ideas as in escaping from old ones.

John Maynard Keynes

\section{Introdução}

Porter e Taisberg ${ }^{1,2}$ referem que a prestação de cuidados de saúde está em rota de colisão com aquilo que são as necessidades dos doentes e a realidade econômica. Aumento de custos, aumento dos problemas de qualidade e um aumento crescente de cidadãos sem acesso aos cuidados de saúde necessários é inaceitável e insustentável, e recomenda estratégias de reforma que permitam obter para os cidadãos melhores cuidados de saúde, para os profissionais a recompensa pelas boas práticas e para os financiadores contenção de custos.

A Organização Mundial da Saúde ${ }^{3}$ refere que também na Europa é necessário um maior investimento na atenção primária para permitir que os sistemas de saúde cumpram todo o seu potencial em benefício dos doentes. Outras organizações e autores ${ }^{4-6}$ partilham esse interesse renovado pela Atenção Primária à Saúde (APS) e dizem que o investimento tem a ver não só com recursos humanos e infraestruturas, mas também com formação, educação, investigação e melhoria contínua da qualidade.

Em outubro de 2005, Portugal iniciou uma ambiciosa reforma da APS, sendo objetivo deste artigo descrever e analisar esse processo de reforma no período de outubro de 2005 a abril de 2010, período em que a Missão para os Cuidados de Saúde Primários (MCSP) teve a responsabilidade de conduzir essa profunda reconfiguração da Atenção Primária Portuguesa.

A metodologia empregue foi um estudo de caso a partir de fontes documentais e observação participante, da perspectiva de gestor governamental atuante em todas as fases da reforma desde o seu desenho à sua implementação no terreno.

É necessário avaliar o desempenho dos sistemas de saúde - e as suas reformas - tendo em conta os principais objectivos da politica de saúde. A reforma foi analisada considerando a institucionalidade, a agenda da reforma e alguns impactos tomando como lógica para a analisar as propostas da OCDE referidas nas lições apreendidas.

\section{Contexto de partida}

Portugal está situado na Península Ibérica, no sudoeste da Europa. É uma nação independente desde 1143, uma república desde 1910, uma democracia desde 1974 e integrou a União Europeia em 1986. Tem centros de saúde comunitários desde 1971, um Serviço Nacional de Saúde desde 1979 e iniciou uma reforma da atenção primária em 2005.

Nos últimos anos, a situação econômica e social em Portugal tem tido um progresso significativo, e a melhoria sistemática dos nossos serviços de saúde tem sido internacionalmente reconhecida ${ }^{7,8}$.

O sistema de saúde português oferece cobertura universal, uma gama completa de serviços de saúde, sendo financiado principalmente através de impostos e apoiando-se sobretudo na prestação pública de cuidados.

Actualmente, temos centros de saúde, hospitais, equipamentos e profissionais de elevada qualidade. A nossa população está cada vez mais consciente dos seus direitos, mais exigente e atenta à proteção da sua saúde ${ }^{9,10}$. No entanto, nem sempre foi assim. Não devemos esquecer as décadas em que a maioria da população não tinha acesso a cuidados de saúde e o sistema de saúde era muito frágil.

No início dos anos 70, Portugal foi um dos primeiros países europeus a adotar uma abordagem integrada para a atenção primária criando uma rede de centros de saúde que abrange a generalidade do país. Os centros de saúde são em Portugal um patrimônio cultural, técnico e institucional que importa não apenas preservar, mas também modernizar e desenvolver pois continuam a ser o meio mais eficiente e acessível para proteger e promover a saúde da população.

São conhecidas as suas limitações e necessidades nomeadamente a crônica carência de recursos humanos, de que os Médicos de Família são apenas a face mais conhecida ${ }^{9,11}$. Precisamos ainda de enfermeiros de família, nutricionistas, psicólogos, assistentes sociais e dentistas, entre outros profissionais. Precisamos aumentar a nossa capacidade resolutiva e dar resposta aos problemas de saúde que diariamente nos apresentam ${ }^{12}$.

Todos os residentes em Portugal têm acesso aos cuidados de saúde disponibilizados pelo Serviço Nacional de Saúde (SNS), estando garanti- 
do o direito de todos os cidadãos à proteção da saúde, a cuidados gratuitos e universais, independentemente do estatuto econômico e social.

A prestação pública está particularmente presente na atenção primária, o primeiro ponto de contacto dentro do sistema público, e nos cuidados hospitalares. Os produtos farmacêuticos, os meios complementares de diagnóstico e as consultas médicas constituem a parte mais significativa da prestação privada de cuidados de saúde ${ }^{13}$.

Os portugueses têm se beneficiado de melhorias substanciais no seu estado de saúde ao longo dos últimos anos. A esperança de vida à nascença (Tabela 1) praticamente duplicou durante o século XX, tanto para as mulheres como para os homens. Esta tendência tem continuado a evoluir favoravelmente, fazendo a esperança de vida convergir para a média da União Europeia ${ }^{15}$. As principais causas de morte devem-se a neoplasias malignas e a patologia cardiovascular.

Os indicadores de saúde infantil (Tabela 1) ainda que melhorando desde o início dos anos sessenta, sofreram reduções excepcionais desde a revolução de 1974. A taxa de mortalidade infantil baixou cinco vezes entre 1970 e 1990, e para menos de metade entre 1991 e 2006, situando-se abaixo do valor médio verificado na UE. Deixaram de estar entre os piores nas décadas de 1980 e 1990 para estar entre os melhores a partir de $2003^{7,13}$.

No entanto, apesar dessas notáveis melhorias, ainda há importantes desigualdades na saúde - entre homens e mulheres, entre regiões e grupos socioeconômicos, e os principais indicadores de desempenho do sistema de saúde ainda não chegaram ao nível da UE ou à média da OCDE. Além disso, os gastos com a saúde passaram de 8,0\% do produto interno bruto em 1996 para $10,2 \%$ em $2008^{15}$, valor que ultrapassa a média da UE, levantando a questão da rentabilidade e sustentabilidade financeira do sistema de saúde ${ }^{16}$. O financiamento público, como percentagem do financiamento total é de cerca de $72 \%$.
O sistema de saúde tem estado em foco no debate político. Apesar de melhorias na saúde das populações, uma preocupação crescente com os níveis de despesa e a consciência cada vez mais clara do desperdício na utilização dos recursos deram origem a várias decisões politicas.

Em 2008, Portugal subscreveu a Carta de Tallinn da $\mathrm{OMS}^{17}$ visando melhorar a saúde das pessoas através do reforço dos sistemas de saúde. Comprometeu-se na promoção da transparência e ser responsável pelo desempenho do sistema de saúde para alcançar resultados mensuráveis. Pediu à OMS uma avaliação da implementação do Plano Nacional de Saúde e do desempenho do sistema de saúde, tendo sido recentemente disponibilizadas as principais conclusões ${ }^{18}$.

\section{Descrição do problema}

Dados estatísticos divulgados pela Direcção Geral da Saúde ${ }^{19}$ relativos a 2006, em nível nacional os cuidados de atenção primária são prestados: (1) em 347 CS, 1.823 extensões, 259 serviços de atendimento permanente (SAP), 41 centros de saúde com internamento num total de 573 camas; (2) por 6.235 especialistas de medicina geral e familiar, por 861 clínicos gerais (não especialistas), por 7.236 enfermeiros e 6.688 administrativos.

Nos centros de saúde estão inscritas 10.110.271 pessoas, das quais $10,6 \%$ não têm médico de família; são utilizadores efetivos 7.435.468, que correspondem a uma taxa de utilização de cerca de 70\%, e foi efetuado um total de 33.997.624.

Segundo o relatório 20 "Análise dos Custos dos Centros de Saúde e do Regime Remuneratório Experimental”, elaborado, a solicitação da MCSP, por um Grupo de Trabalho da Associação Portuguesa de Economia da Saúde coordenado pelo Prof. Miguel Gouveia, resumido na Tabela 2, os centros de saúde são demasiado grandes para a

Tabela 1. Evolução de indicadores relevantes.

\begin{tabular}{lcccc}
\hline & $\mathbf{1 9 2 0}$ & $\mathbf{1 9 8 0}$ & $\mathbf{2 0 0 0}$ & $\mathbf{2 0 0 7}$ \\
\hline Esperança de vida à nascença, em anos, mulheres & 40,0 & 74,6 & 80,3 & 82,0 \\
Esperança de vida à nascença, em anos, homens & 35,8 & 67,5 & 73,2 & $\mathbf{7 5 , 5}$ \\
\hline & $\mathbf{1 9 8 0}$ & $\mathbf{1 9 9 0}$ & $\mathbf{2 0 0 0}$ & $\mathbf{2 0 0 8}$ \\
\hline Taxa de mortalidade infantil por 1.000 nascidos vivos & 24,3 & 11,0 & 5,54 & 3,3 \\
\hline
\end{tabular}

Fonte: Barros e Simões ${ }^{13}$, Organisation for Economic Co-operation and Development (OECD $)^{14}$. 
prestação de cuidados, tendo em conta a prestação de serviços com proximidade e qualidade, e demasiado pequenos para efeitos de economias de escala, obtenção de ganhos de eficiência e melhorias da gestão.

Embora em teoria o sistema de saúde pareça bom, na realidade continua a enfrentar múltiplos problemas ${ }^{13,21}$. O SNS é uma estrutura altamente centralizada e burocrática, não existindo incentivos para o bom desempenho ou para a qualidade. A insatisfação profissional é um desafio e uma ameaça para o desenvolvimento da Atenção Primária. Médicos e enfermeiros são funcionários públicos com salários fixos independentes do desempenho ${ }^{22}$.

O ponto de partida para a actual reforma assenta pois num baixo nível de satisfação de todos os intervenientes, cidadãos, profissionais e decisores políticos, motivado pela baixa acessibilidade, ineficiência, barreiras burocráticas e falta de incentivos para melhorar a produtividade e a qualidade.

\section{Desenvolvimento do Projeto e soluções encontradas}

O governo procura organizar o sistema de saúde de molde a melhorar a justiça distributiva, a eficiência, a efetividade e a capacidade de resolver os problemas dos cidadãos. Todos os esforços são feitos para manter os princípios de solidariedade e justiça, para que todos possam se beneficiar dos avanços científicos e tecnológicos postos ao serviço da saúde e do bem-estar.

Para concretizar esta visão, prestar melhores cuidados e obter mais saúde, tornou-se necessário mudar o cenário da atenção primária, tornando-a mais acessível, mais eficiente e mais adequada às necessidades, não apenas em resposta às necessidades dos cidadãos, mas também dos profissionais.

O Programa do XVII Governo Constitucional, na área da saúde, dava um particular enfoque à atenção primária e à sua importância na ligação ao usuário, por ser o primeiro acesso deste aos cuidados de saúde.

Referia explicitamente que a atenção primária é o pilar central do sistema de saúde, que o Centro de Saúde (CS) constitui a entidade enquadradora das unidades de saúde familiar (USF) e que seriam adoptadas as seguintes medidas de política:

. Reestruturação dos CS através da criação de USF, obedecendo aos princípios seguintes: (1) pequenas equipas multiprofissionais e auto-organizadas; (2) autonomia organizativa funcional e técnica; (3) contratualização de uma carteira básica de serviços; (4) meios de diagnósticos descentralizados; (5) sistema retributivo que premeie a produtividade, acessibilidade e qualidade; . As USF devem ser integradas, em rede, podendo assumir diferentes enquadramentos jurídicos na sua gestão, quer pertencendo ao sector

Tabela 2. Análise da produção e custos dos Centros de Saúde em Portugal em 2005.

\begin{tabular}{|c|c|}
\hline $\begin{array}{l}\text { Dimensão e produção anual } \\
\text { de um Centro de Saúde médio }\end{array}$ & $\begin{array}{l}\text { Custo médio anual } \\
\text { por paciente registrado: } € 216,5\end{array}$ \\
\hline total pacientes registrados: 30.834 & . vencimentos dos médicos: $€ 34,8$ \\
\hline sem médico de família: $10,8 \%$ & . vencimentos de enfermeiros: $€ 14,0$ \\
\hline . usuários utilizadores num ano: 19.792 & . custos administrativos e direcção: $€ 18,6$ \\
\hline médicos: 20,9 & . custo anual médio com exames \\
\hline . enfermeiros: 20,6 & complementares de diagnóstico: $€ 40,9$ \\
\hline consultas num ano: 97.315 & . custo anual médio medicamentos: $€ 84,3$ \\
\hline . Custos totais anuais: $€ 6.674 .690$ & . outros custos: $€ 24$ \\
\hline $\begin{array}{l}\text { Custo médio anual por usuário utilizador } \\
\text { efectivo: } € 337,3\end{array}$ & Custo por consulta médica: $€ 68,6$ \\
\hline . vencimentos médicos: $€ 54,2$ & . vencimento do médico: $€ 11,0$ \\
\hline $\begin{array}{l}\text { custo anual médio com exames complementares } \\
\text { de diagnóstico: } € 63,7\end{array}$ & $\begin{array}{l}\text {. custo com exames complementares de } \\
\text { diagnóstico: } € 13,0\end{array}$ \\
\hline custo anual médio medicamentos: $€ 131,4$ & . custo com medicamentos: $€ 26,7$ \\
\hline
\end{tabular}

Fonte: Prof. Miguel Gouveia, Grupo de Trabalho da APES. 
público administrativo, quer pertencendo ao sector cooperativo, social e privado;

. Reforçar os incentivos e a formação dos médicos de família, atraindo jovens candidatos.

$\mathrm{Na}$ sequência do estabelecido no Programa do Governo, o Conselho de Ministros ${ }^{23}$ criou na dependência direta do Ministro da Saúde a Missão para os Cuidados de Saúde Primários (MCSP), com a natureza de estrutura de missão, para a condução do projecto global de lançamento, coordenação e acompanhamento da estratégia de reconfiguração dos CS e implementação das USF.

Nessa resolução a MCSP é incumbida, entre outras tarefas, de:

. Apoiar a reconfiguração dos CS em USF, desempenhando um papel de provedoria das iniciativas dos profissionais;

. Coordenar tecnicamente o processo global de lançamento e implementação das USF, bem como dos demais aspectos de reconfiguração dos CS;

. Desempenhar funções de natureza avaliadora, reguladora de conflitos e de apoio efetivo às candidaturas das USF;

. Propor a orientação estratégica e técnica sobre a política de recursos humanos, a formação contínua dos profissionais e a política de incentivos ao desempenho e à qualidade, a aplicar nas USF;

. Elaborar os termos de referência da contratualização das ARS com os CS e destes com as USF;

. Promover o lançamento de formas inovadoras de melhoria da articulação com outras unidades de prestação de cuidados, nomeadamente com os cuidados hospitalares e continuados;

- Propor, nos termos da lei, modalidades de participação dos municípios, cooperativas, entidades sociais e privadas na gestão de CS e USF.

Por Resolução do Conselho de Ministros, o mandato da MCSP foi prorrogado por dois anos e posteriormente mais um ano. A MCSP terminou, em 14 de abril de 2010, o seu mandato, após quatro anos e meio de actividade.

Em janeiro de 2006, a MCSP publica as "Linhas de Acção Prioritária para o Desenvolvimento dos Cuidados de Saúde Primários" ${ }^{24}$, em que divulga as suas propostas de medidas em oito áreas de actuação e clarifica qual seria a evolução da reforma.

Estabeleceu como grandes objetivos para a Reforma da Atenção Primária a obtenção de mais e melhores cuidados de saúde para os cidadãos, aumento da acessibilidade e consequente aumento da satisfação dos utilizadores dos serviços. Preten- deu-se também aumentar a satisfação dos profissionais criando boas condições de trabalho, melhorando a organização e recompensando as boas práticas. Simultaneamente, tem que se melhorar a eficiência e promover a contenção de custos.

A reforma da atenção primária insere-se no quadro mais vasto da reforma da Administração Pública, passa pela reconfiguração dos CS obedecendo a um duplo movimento: (1) a constituição de pequenas unidades funcionais autónomas - as USF - prestadoras de cuidados de saúde à população; (2) a criação de agrupamentos de CS, promovendo a agregação de recursos e estruturas de gestão, eliminando concorrências estruturais, obtendo economias de escala.

\section{Unidades de Saúde Familiar}

A fase inicial, e com maior visibilidade pública, foi a criação de USF, pequenas equipas multiprofissionais, voluntárias, auto-organizadas e compostas por três a oito médicos de família, igual número de enfermeiro de família e secretários clínicos, abrangendo uma população entre 4.000 e 14.000 pessoas. Estas equipas têm autonomia técnica, funcional e organizativa e muito importante, um sistema de pagamento misto ( $\mathrm{ca}$ pitação, salário, objectivos), incentivos financeiros e profissionais que recompensam o mérito (baseado em actividades) e é sensível à produtividade e acessibilidade, mas também, e acima de tudo, à qualidade ${ }^{25,26}$.

A reestruturação também está em andamento na reorganização da intervenção comunitária, uma melhoria dos cuidados domiciliários, melhoria dos cuidados paliativos, a utilização de unidades móveis em certas situações e a utilização de redes de apoio às famílias.

\section{Agrupamentos de Centros de Saúde}

A reconfiguração dos CS e a criação de ACES tem o objectivo principal de dotar os actuais CS dum enquadramento organizacional e de estruturas de apoio, que lhes permitam aumentar a qualidade dos serviços prestados, maximizando os recursos e preservando a sua identidade, passando de uma lógica de tutela para uma lógica de prestação de serviços e optimização dos fluxos de informação.

Esses agrupamentos, num máximo de setenta e quatro em nível nacional, devem obedecer, em regra, a um critério populacional que corresponda a um número de pessoas residentes entre 50 a 200 mil e complementado por um conjunto 
de variáveis como a acessibilidade geográfica, a densidade populacional e a acessibilidade das pessoas a cuidados hospitalares.

Os CS, reorganizados no novo modelo de ACES, caracterizam-se por $^{27}$ uma estrutura organizacional assente em cinco tipos de unidades funcionais descritas no Quadro 1, com trabalho em equipa multiprofissional, com missões específicas, intercooperantes e complementares, organizadas em rede, têm autonomia administrativa para decidir e implementar soluções adaptadas aos recursos e às condições de cada local e comunidade, órgãos e instrumentos próprios de gestão organizacional, sistemas de liderança e de governação clínica e técnica bem definidos, mecanismos de representação e de participação da comunidade e dos cidadãos.

É celebrado um contrato-programa entre o director executivo do ACES e o conselho directivo da Administração Regional de Saúde pelo qual se estabelecem, qualitativa e quantitativamente, os objectivos do ACES e os recursos afectados ao seu cumprimento e se fixam as regras relativas à respectiva execução.

Quadro 1. Agrupamentos de Centros de Saúde. Estrutura orgânica e unidades funcionais.

ACES - Agrupamento de Centros de Saúde - Nova matriz organizacional e funcional assente em unidades funcionais com autonomia gestionária, constituídas por equipas multiprofissionais, funcionamento em rede e compromissos assistenciais contratualizados.

\begin{tabular}{|c|c|}
\hline USF & $\begin{array}{l}\text { Unidade de Saúde Familiar - Cuidados de saúde à pessoa e à família. Unidade que } \\
\text { promove a formação de equipas multidisciplinares para o desempenho de cuidados } \\
\text { personalizados. As USF distinguem-se das UCSP pelo nível de desenvolvimento e de dinâmica } \\
\text { de equipa, designadamente pelos compromissos de cooperação interprofissional livremente } \\
\text { assumidos. Está regulamentada em legislação própria. }\end{array}$ \\
\hline UCSP & $\begin{array}{l}\text { Unidade de Cuidados de Saúde Personalizados - Cuidados de saúde à pessoa e à família. } \\
\text { Tem dimensão idêntica à prevista para as USF e presta cuidados personalizados, garantindo a } \\
\text { acessibilidade, a continuidade e a globalidade dos mesmos, sendo constituída por equipa } \\
\text { multiprofissional composta por médicos, enfermeiros e administrativos não integrados em } \\
\text { USF. Depende hierarquicamente do Director Executivo e está vinculada às normas aprovadas } \\
\text { pelo Conselho Clínico em matéria de governação clínica. }\end{array}$ \\
\hline UCC & $\begin{array}{l}\text { Unidade de Cuidados na Comunidade - Cuidados organizados e orientados para grupos } \\
\text { e ambientes específicos. Tem actuação multidisciplinar, que engloba a prestação de cuidados } \\
\text { de saúde e de apoio psicológico e social, de base geográfica e domiciliária, designadamente na } \\
\text { identificação e acompanhamento de indivíduos e famílias de maior risco, dependência e } \\
\text { vulnerabilidade de saúde. }\end{array}$ \\
\hline USP & $\begin{array}{l}\text { Unidade de Saúde Pública - Saúde populacional, ambiental e pública. Coordenam } \\
\text { intervenções orientadas para garantir o bem público comum no domínio da saúde. } \\
\text { Funcionam como observatórios de saúde local, unidade de administração de saúde } \\
\text { populacional, de coordenação de estratégias locais de saúde de âmbito comunitário. } \\
\text { Responsável pela realização de estudos populacionais, vigilância epidemiológica e exercício da } \\
\text { função de autoridade de saúde. }\end{array}$ \\
\hline URAP & $\begin{array}{l}\text { Unidade de Recursos Assistenciais Partilhados - Apoio técnico-assistencial a todas as } \\
\text { unidades. É uma unidade que organiza e coordena múltiplos meios, recursos e competências } \\
\text { assistenciais específicas de cada ACES (higiene oral, fisioterapia, terapia da fala, terapia } \\
\text { ocupacional, psicologia, serviço social, nutricionismo e outros profissionais não afectos } \\
\text { totalmente a outras unidades funcionais) cuja missão é a de apoiar as demais unidades } \\
\text { funcionais. }\end{array}$ \\
\hline \multicolumn{2}{|r|}{$\begin{array}{l}\text { UAG - Unidade de Apoio à Gestão - Apoio logístico ao funcionamento de todas as equipas e dos órgãos } \\
\text { de gestão. Viabilização do funcionamento adequado de toda a organização. A UAG é uma unidade de } \\
\text { back-office que viabiliza que, em cada momento, existam condições materiais e objectivas para que todos } \\
\text { possam cumprir a sua missão. }\end{array}$} \\
\hline
\end{tabular}




\section{Governação clínica}

Uma das inovações do novo modelo de organização foi a criação de uma hierarquia técnica, instituída através da formação de um Conselho Clínico, cujo presidente é o Director Clínico, obrigatoriamente um Médico de Família. Integram ainda o Conselho Clínico um Enfermeiro, um Médico de Saúde Pública e uma outra profissão como, por exemplo, um nutricionista ou psicólogo. Entre as suas atribuições ${ }^{27}$ salientaria a avaliação da efectividades dos cuidados de saúde prestados, fixar procedimentos que garantam a melhoria contínua da qualidade dos cuidados de saúde, nomeadamente aprovando orientações clínicas relativas à prescrição de medicamentos $\mathrm{e}$ meios complementares de diagnóstico e terapêutica, assim como protocolos clínicos adequados às patologias mais frequentes. Apoiam o director executivo em assuntos de natureza técnicoprofissional e de gestão clínica, decidem sobre conflitos de natureza técnica, monitorizam o grau de satisfação dos profissionais e organizam as atividades de desenvolvimento profissional contínuo e de investigação.

\section{Formação de dirigentes}

Os ACES têm que se integrar numa lógica de organizações flexíveis com capacidade de adaptação. Deles se espera um novo quadro de gestão descentralizada, criando os mecanismos que lhes permitam fazer de forma cíclica uma correcta avaliação das necessidades, planeamento estratégico, olhar para a prestação de cuidados e adequá-la às necessidades da população, avaliar e monitorizar o desempenho. Para se desenvolver uma mudança organizacional efectiva houve a preocupação de que os Directores Executivos tivessem acesso a formação específica, direcionada para as funções que iriam desempenhar. Tratou-se de uma iniciativa inédita e inovadora na Administração Pública Portuguesa.

O PACES-Programa Avançado em Gestão, Governação Clínica, Liderança e Sistemas de Informação para dirigentes dos $\mathrm{ACES}^{28}$ é um projeto de formação inovador, com o objectivo essencial de criar uma cultura e prática de gestão que resultam de aprendizagens e vivências partilhadas. Foi coordenado pelo Instituto Nacional de Administração (INA), que é uma instituição criada para valorizar e qualificar os recursos humanos da Administração Pública.

O PACES pretendia fornecer as ferramentas fundamentais de gestão, numa dinâmica de for- mação-ação, favorecendo práticas de inovação e sobretudo criar uma rede de colaboração entre diretores executivos aproveitando o fato de terem sido nomeados simultaneamente setenta e quatro altos dirigentes da atenção primária. O programa para directores executivos teve a duração de doze meses e 400 horas de formação. O programa TEAM para os Conselhos Clínicos abrangeu todos os membros dos setenta e quatro ACES. O TEAM foca-se na criação de uma cultura de equipa nos vários conselhos clínicos e o objetivo é criar uma rede de aprendizagem e partilha, um espaço de reflexão, para encontrar soluções, trocar experiências e ideias, e sobretudo potenciar uma nova cultura de governação clínica.

É uma iniciativa inovadora e que muito contribuirá para a implementação com sucesso dos ACES e das suas unidades funcionais e que tem continuação com programas de formação para as USP que já se iniciaram e para as UAG a iniciar brevemente.

Em resumo, o actual processo de mudança passou pela:

. Constituição das USF;

. Criação dos ACES;

. Introdução de novo modelo de gestão;

. Instituição de governação clínica;

- Reorganização dos serviços de suporte.

Para levar por diante este desafio, a MCSP elaborou um plano estratégico ${ }^{29}$ constituído por dez linhas estratégicas, agrupadas em três eixos, contemplando um vasto conjunto de objectivos que correspondem aos resultados que se pretendia ver alcançados no final desta primeira fase da reforma.

\section{Resultados obtidos}

\section{Legislação}

Foi publicado, em agosto de 2007, o decretolei que estabeleceu o regime jurídico da organização e funcionamento aplicável a todos os modelos de USF, assim como o regime de incentivos a atribuir a todos os elementos que as constituem, bem como a remuneração a atribuir aos elementos que integrem as USF de modelo $\mathrm{B}^{25}$.

Em fevereiro de 2008 foi publicado o decretolei ${ }^{27}$ que estabeleceu o regime jurídico da organização e funcionamento dos ACES, bem como toda a legislação relativa às suas unidades funcionais. O quadro legislativo da Reforma está completo. 


\section{Unidades funcionais}

As USF estão todas informatizadas, têm um conjunto de indicadores de desempenho para a sua avaliação ${ }^{30}$, contratualizam objetivos ${ }^{31,32} \mathrm{e}$ para efeito de pagamento dos incentivos institucionais são avaliados e monitorizados indicadores em sete áreas: disponibilidade, acessibilidade, produtividade, qualidade técnico-científica, efectividade, eficiência e satisfação.

Os indicadores associados ao pagamento de incentivos financeiros aos médicos para além dos cuidados domiciliários integram a vigilância de planeamento familiar, vigilância da gravidez, seguimento da criança no $1^{\circ}$ e $2^{\circ}$ ano de vida, vigilância da diabetes e vigilância da hipertensão.

No final de abril de 2010, quarenta e quatro meses após a abertura das primeiras dez USF em Setembro de 2006, estavam em funcionamento 242 unidades (Gráfico 1), numa média de 5,5 aberturas por mês, das quais 102 em Modelo B (com pagamento ligado ao desempenho) abrangendo (Gráfico 2) 4.836 profissionais, dando cobertura a 3.043.044 cidadãos (Gráfico 3). A média das listas anteriores era de cerca de 1.550 pessoas e actualmente a média é de 1.770 por médico. Com este aumento das listas foi possível dar médico de família a 368.021 pessoas, o que representa um acréscimo de cobertura de $13,7 \%$. A média de inscritos por USF é de 12.600 pessoas, para um total de 20 profissionais dos quais sete médicos. Cento e dez candidaturas estão ativas aguardando a sua entrada em funcionamento.

Os ACES foram todos criados e as suas unidades funcionais vão sendo sucessivamente co-

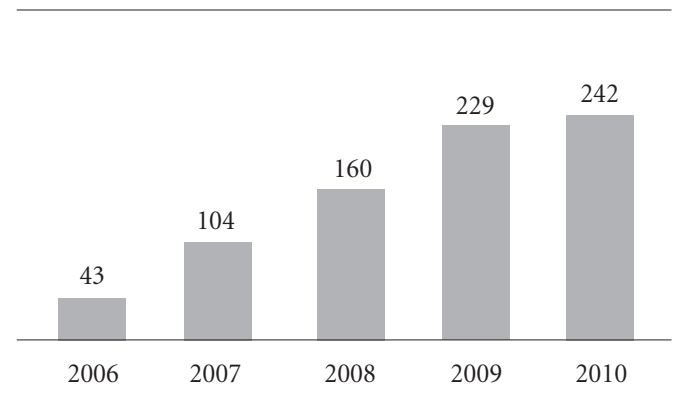

Gráfico 1. Número de USF em atividade por ano em Portugal entre setembro de 2006 e abril de 2010 .

Fonte: Missão para os Cuidados de Saúde Primários. locadas no terreno. Os seus dirigentes foram todos nomeados e participaram em acões de formação especificamente desenhadas para eles.

\section{Ganhos de eficiência}

Está a ser ultimado um estudo, resultante de uma tese de doutoramento sobre a eficiência das diferentes unidades existentes actualmente na atenção primária.

Pode-se, no entanto, desde já, verificar um aumento dos níveis de eficiência nos relatórios dos departamentos de contratualização. Por exemplo, em média, cada USF do norte do país gastou cerca de $170 €$ em medicamentos e $65 € \mathrm{em}$ ECD (contra $204 €$ em medicamentos e $95 €$ com ECD nos centros de saúde de origem) por utilizador/ano, resultando na poupança de muitos milhares de euros - menos $21,9 \%$ nos medicamentos e $27,0 \%$ nos MCDT $^{32}$. De salientar que as taxas de cobertura são sistematicamente maiores que nos CS da mesma região.

\section{Satisfação de usuários e profissionais}

A MCSP encomendou um estudo a uma entidade independente (Centro de Estudos e Investigação em Saúde, da Faculdade de Economia da Universidade de Coimbra) sobre a monitorização da satisfação dos profissionais e dos usuários de todas as 146 USF constituídas até novembro de 2008. O estudo foi publicado em livro ${ }^{33}$ e está disponível nas páginas web da MCSP e do CEISUC ${ }^{34}$.

$\mathrm{Na}$ sequência de um censo de um dia, foram entregues 16.768 questionários com uma taxa

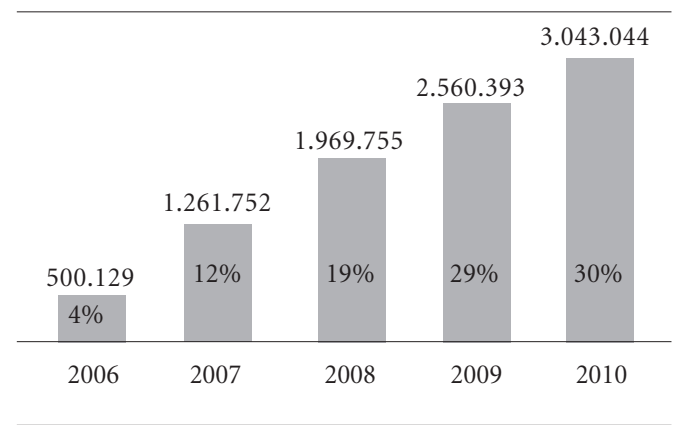

Gráfico 2. População inscrita em USF em Portugal, por ano, em valor absoluto e percentagem da população total entre setembro de 2006 e abril de 2010.

Fonte: Missão para os Cuidados de Saúde Primários. 
Gráfico 3. Profissionais de saúde a trabalhar em USF em Portugal, por ano e por profissão, entre setembro de 2006 e abril de 2010.

Fonte: Missão para os Cuidados de Saúde Primários.

global de respostas de $75,8 \%$. Constatou se a existência de satisfação com os cuidados prestados pelos profissionais destas USF, realçando se as componentes relacionais e humanas. Detectou se, por outro lado, uma menos alta satisfação em relação à organização dos serviços, nomeadamente em relação aos tempos de espera que os utilizadores continuam a sentir ser exagerados.

Verificou se, em geral, a existência de um grande apreço pela competência, pelo carinho e pela cortesia dos prestadores de cuidados, pelo respeito com que os utilizadores sentem ser tratados e pelas condições das instalações. Os serviços ao domicílio atingem, ainda, no entanto, valores menos bons neste contexto.

A análise qualitativa permitiu também aprofundar e complexificar a informação obtida através dos dados quantitativos, dando voz às palavras dos inquiridos. Os textos dos comentários e opiniões revelaram uma preponderância das surpresas agradáveis sobre as desagradáveis no contacto com as USF, assim como uma valorização dos aspectos relacionais dos cuidados e das qualidades e desempenho dos profissionais, confirmando a análise da informação quantitativa relativa à satisfação.

Rapidamente, as USF se tornaram unidades de saúde com uma certa autonomia, responsá- veis pela saúde, bem-estar e qualidade de vida da população servida. Os resultados obtidos pelo presente estudo devem ser encarados com ânimo e esperança na sustentabilidade deste processo de reforma.

O estudo sobre a monitorização da satisfação dos profissionais das USF $^{35}$ pretende monitorizar a avaliação que os profissionais das USF fazem do local de trabalho e dos cuidados aí prestados. Foi feita uma sondagem pela Internet a todos os profissionais das USF constituídas até novembro de 2008. Dos 2.821 profissionais então existentes nas USF em estudo, 2.398 acederam à página concebida pelo CEISUC e responderam ao questionário, o que corresponde a uma taxa de respostas de $85,0 \%$, muito superior ao que é habitual neste tipo de estudos. Como exemplo, podemos salientar que $72,2 \%$ estão satisfeitos com a qualidade da USF como local de trabalho, 73,6\% com a política de recursos humanos, $80,2 \%$ com o estado de espírito, $80,3 \%$ com o superior hierárquico, $48,6 \%$ com o vencimento e $61,4 \%$ com o local de trabalho e equipamento.

Em resumo, as USF conseguiram simultaneamente mais eficiência, mais acessibilidade, melhor clima laboral, maior satisfação dos cidadãos, numa palavra, mais qualidade. Constituem um bom exemplo do sucesso da filosofia de 
gestão que recomenda não dizer às pessoas como fazer as coisas. Atribuir uma missão para cumprir e dar liberdade para o fazer. Esta solução organizativa despertou algum interesse em nível nacional e internacional ${ }^{36-38}$.

\section{Lições aprendidas}

Por vezes, assegura-se que o conteúdo de uma reforma é menos importante na sua aprovação pública e legislativa que o timing da sua apresentação, a forma como é apresentada e discutida com as partes interessadas, e uma infinidade de outros factores. A Organização para a Cooperação e Desenvolvimento Económico (OCDE) está a desenvolver um projecto de caráter transversal sobre estas questões, chamado "Fazer a reforma acontecer"39.

Estão a ser estudados os fatores por trás da implementação de reformas bem- sucedidas, com base em evidências provenientes de países que viram nos últimos anos os seus sistemas de saúde analisados pela OCDE e sobre uma exaustiva revisão da literatura. O projeto considera quatro questões em particular, utilizadas em todas as diferentes áreas estudadas:

- A existência de instituições adequadas para apoiar as reformas da decisão à implementação.

. O impacto e as reações dos afectados pelas reformas.

- A agenda da reforma, calendário e as interações entre as diferentes áreas.

- O papel das evidências e das organizações internacionais no apoio às reformas.

Conclui-se que algumas fases da reforma têm que estar concluídas antes de se poder dizer que uma reforma foi bem-sucedida; uma falha numa delas geralmente leva ao fracasso da reforma. Questões específicas do setor da saúde incluem entre outras o papel dos grupos profissionais que prestam serviços de saúde, da informação, das evidências disponíveis, das comparações internacionais no desempenho dos sistemas de saúde, de um diagnóstico claro e um design atraente para a reforma, o aproveitamento das "janelas de oportunidade" políticas, a utilização de incentivos, para alinhar os interesses das partes interessadas com as intenções da reforma e assegurar recursos suficientes para "lubrificar as engrenagens da mudança”.

É necessário avaliar o desempenho dos sistemas de saúde - e as suas reformas - tendo em conta os principais objectivos da política de saú- de. O trabalho da OCDE tem-se focado preferencialmente no acesso, capacidade de resposta, sustentabilidade, qualidade, equidade e eficiência.

Salientamos como importante para o sucesso da reforma o fato de a criação das USF ser um processo voluntário da base para o topo, com total envolvimento dos profissionais de saúde. Estes fizeram a escolha da equipa, definiram um plano de ação, têm autonomia organizacional e de gestão, em função de objectivos, e corresponsabilização.

Também importante foi a existência de um forte apoio político e da existência de uma boa interacção com a comunicação social.

Finalmente, a importância da reforma se centrar na melhoria da acessibilidade dos utilizadores aos serviços e na melhoria da qualidade do atendimento.

O desaparecimento da MCSP, concluída que foi a fase de planeamento, arranque e implementação das vertentes e componentes essenciais, abriu caminho a uma segunda etapa de desenvolvimento com a institucionalização e internalização dos dispositivos de prossecução da reforma, trazendo alguma apreensão sobre a sua sustentabilidade.

A abertura de novas USF e o ultrapassar de mais de $50 \%$ de cobertura por este novo modelo organizativo está de algum modo assegurada pelas mais de uma centena de candidaturas em carteira. Mas a sustentabilidade da reforma está muito ligada ao sucesso no combate à crescente $\mathrm{e}$ ameaçadora falta de recursos humanos, principalmente médicos de família, ultrapassar as conhecidas insuficiências do sistema de informação, conseguir colocar as unidades funcionais dos ACES a trabalhar em rede, criar mecanismos mais efectivos de acompanhamento e avaliação. Mas acima de tudo, o maior risco para a sustentabilidade de qualquer reforma tem a ver com as alterações nas prioridades políticas e um eventual desinvestimento político.

Segundo Pedro Ferreira ${ }^{33}$, a reforma dos CSP em curso, consubstanciada inicialmente pela aplicação de novas medidas de gestão e pela criação das USF, foi possível graças a um feliz encontro de três determinantes fundamentais: a vontade dos profissionais e a sua aposta na mudança, a liderança profissional conduzida por um grupo de missão e, por fim, o constante apoio político por parte do Ministério da Saúde. A ausência de qualquer um destes pilares de desenvolvimento teria inviabilizado todo o processo. 


\section{Referências}

1. Porter M, Teisberg E. Redefining health care: creating value-based competition on results. Cambridge, MA: Harvard Business School Press; 2006.

2. Porter M, Teisberg E. How physicians can change the future of health care. JAMA 2007; 297(10):11031111.

3. Atun R. What are the advantages and disadvantages of restructuring a health care system to be more focused on primary care services? Health Evidence Network Report (HEN). Copenhagen: WHO Regional Office for Europe; 2004.

4. Word Health Organization (WHO). Relatório Mundial de Saúde 2008. Cuidados de Saúde Primários Agora Mais Que Nunca. Geneve:WHO; 2008. [documento na Internet]. [acedido 2010 jul. 10]. Disponível em: http://www.who.int/whr/2008/whr08_ pr.pdf

5. Saltman RB, Rico A, Boerma WGW, eds. Primary care in the driver's seat? Organizational reform in European primary care. Maidenhead: Open University Press/McGraw-Hill Education; 2006. [documento na Internet]. [acedido 2010 jul. 10]. Disponível em: http://whqlibdoc.who.int/euro/2006/ 0335213650_eng.pdf

6. Barbara Starfield. Refocusing the System. N Engl J Med 2008; 359(20):2087-2091.

7. Bentes M, Dias CM, Sakellarides C, Bankauskaite V. Health care systems in transition: Portugal. Copenhagen: WHO Regional Office for Europe on behalf of the European Observatory on Health Systems and Policies; 2004. [documento na Internet]. [acedido 2010 jul. 10]. Disponível em: http://www.euro. who.int/document/e82937.pdf

8. Organization for Economic Co-operation and Development (OCDE). Health at a glance 2009. Paris: OCDE; 2007. [documento na Internet]. [acedido 2010 jul. 10]. Disponível em: http://www.oecd.org/ health/healthataglance

9. Pisco L. Êxitos e insucessos da Medicina Geral e Familiar Portuguesa ou o que conseguimos em 20 anos e o que falta conseguir. In: Pisco L. Da Memória. Lisboa: MVA Invent; 2003. p. 15-23.

10. Santos O, Biscaia A, Antunes AR, Craveiro I, Júnior A, Caldeira R, Charondière P. Os centros de saúde em Portugal: a satisfação dos utentes e dos profissionais. Lisboa: Missão para os Cuidados de Saúde Primários; 2007.

11. Biscaia A, Martins J, Carreira M, Fronteira I , Antunes A, Ferrinho P. Cuidados de Saúde Primários em Portugal: reformar para novos sucessos (Grande Prémio Fundação AstraZeneca 2005). 2a ed. Lisboa: Padrões Culturais Editora; 2008.

12. Pisco L. Cuidados de Saúde Primários: o futuro. O futuro da Saúde em Portugal. Lisboa: Companhia de Ideias; 2007. p.133-142.

13. Barros P, Simões JA. Portugal: Health system review. Health Systems in Transition [periódico en Internet]. 2007; [acedido 2010 jul. 10]; 9(5):1-140. Disponível em: http://www.euro.who.int/_data/ assets/pdf_file/0004/107842/E90670.pdf
14. Organisation for Economic Co-operation and Development (OECD). Health at a glance. Europe 2010. Paris: OCDE; 2010. [ documento na internet]. [acessado 2010 jul 10]. Disponível em: http://www.oecd. org/dataoecd/2/46/46727776.pdf

15. Organization for Economic Co-operation and Development (OCDE) Health Database [online database]. Paris: OECD; 2009. [documento na Internet]. [acedido 2010 jul. 10].Disponível em: http:// www.oecd.org/health/healthdata.

16. Thomson S, Foubister T, Figueras J, Kutzin J, Permanand G, Bryndová L. Addressing financial sustainability in health systems. Copenhagen: WHO Regional Office for Europe on Behalf of the European Observatory on Health Systems and Policies; 2009. [documento na Internet]. [acedido 2010 jul. 10]. Disponível em: http://www.euro.who.int/ __data/assets/pdf_file/0005/64949/E93058.pdf

17. WHO Regional Office for Europe. The Tallinn Charter: Health Systems for Health and Wealth. Copenhagen: WHO Regional Office for Europe; 2008. [documento na Internet]. [acedido 2010 jul. 10]. Disponível em: http://www.euro.who.int/document/ e91438.pdf.

18. WHO Regional Office for Europe. WHO evaluation of the National Health Plan of Portugal (2004-2010). Copenhagen: WHO Regional Office for Europe; 2010. [documento na Internet]. [acedido 2010 jul. 10]. Disponível em: http://www.euro.who.int/ __data/assets/pdf_file/0003/83991/E93701.pdf

19. Portugal. Direcção-Geral da Saúde,editor. Centros de Saúde e Hospitais: Recursos e produção do SNS ano de 2006. Lisboa: Direção-Geral de Saúde; 2007.

20. Gouveia M, Silva NS, Oliveira P, Miguel LS. Análise dos Custos dos Centros de Saúde e do Regime Remuneratório Experimental. Lisboa: Grupo de Trabalho da Associação Portuguesa de Economia da Saúde; 2006.

21. Cabral V, Silva P. O Estado da Saúde em Portugal, Acesso, avaliação e atitudes da população portuguesa em relação ao sistema de saúde. evolução entre 2001 e 2008 e comparações regionais. Lisboa: Imprensa de Ciências Sociais; 2009.

22. Pisco L. Remuneração dos Médicos de Família. Financiamento, Inovação e Sustentabilidade. Lisboa: Companhia de Ideias; 2008. p. 218-230.

23. Resolução do Conselho de Ministros no 157/2005 de 22 de Setembro de 2005. [documento na Internet]. [acedido 2010 jul. 10].Disponível em: http:// mcsp.lvengine.com/Imgs/content/page_12/

24. Resolucao_Conselho_Ministros_157_2005.pdf Resolução do Conselho de Ministros no 157/2005, de 12 de outubro. Linhas de Acção Prioritária para o Desenvolvimento dos Cuidados de Saúde Primários. Missão para os Cuidados de Saúde Primários. Diário da República 2005; I Série B- n. ${ }^{\circ}$ 196. Lisboa: Ministério da Saúde; 2006. [documento na Internet]. [acedido 2010 jul. 10]. Disponível em: http:// www.portugal.gov.pt/pt/Documentos/Governo/MS/ Linhas_Cuidados_Saude_Primarios.pdf 
25. Regime Jurídico das USF. Diário da República 2007; 1. ${ }^{a}$ série - No 161 - DL no 298/2007, de 22 de Agosto de 2007. [documento na Internet]. [acedido 2010 jul. 10]. Disponível em: http://www.min-saude.pt/ NR/rdonlyres/95C24BF5-4850-4C49-AA0BA1BDB23B52BB/0/DL298_2007de22Ago_RegJuridico dasUSF.pdf

26. Indicadores de desempenho para as unidades de saúde familiar. Missão para os Cuidados de Saúde Primários. Lisboa: Minsitério da Saúde; 12 de abril de 2006. [documento na Internet]. [acedido 2010 jul. 10]. Disponível em: URL: http://mcsp.lvengine. com/Imgs/content/page_46/Indicadores_ desempenho_USF_MCSP20060412.pdf

27. Regime Jurídico dos ACES. Diário da República 2008; 1. ${ }^{a}$ série - no 38 - Decreto-Lei n.o $28 / 2008$ de 22 de Fevereiro de 2008. [documento na Internet]. [acedido 2010 jul. 10]. Disponível em: http://www. acs.min-saude.pt/files/2008/02/agrupamento_ centros_saude.pdf [Acedido em 10 Julho 2010].

28. Lapão L, organizador. Programa Avançado de Gestão para Directores Executivos dos ACES :Livro de Curso. Oeiras: INA Editora; 2010.

29. Reforma dos Cuidados de Saúde Primários. Plano Estratégico 2007-2009. Missão para os Cuidados de Saúde Primários. Lisboa; abril 2007. [acedido 2010 jul. 10]. Disponível em: http://www.portugal.gov.pt/ pt/Documentos/Governo/MS/Estrategia_Cuidados_ Saude_Primarios.pdf

30. Matriz de indicadores, a contratualizar com as USF para o ano de 2006 e 2007. Agências de contratualização dos serviços de saúde. Lisboa; 11 de maio de 2006. [acedido 2010 jul. 10]. Disponível em: http:/ /mcsp.lvengine.com/Imgs/content/page_46/ Contratualizacao\%20-\%202007.pdf

31. Contratualização com as unidades de saúde familiar para 2007. Agências de contratualização dos serviços de saúde; 28 de dezembro de 2006. [acedido 2010 jul. 10]. Disponível em: http://mcsp.lvengine. com/Imgs/content/page_46/Contratualizacao $\% 20$ \%202007.pdf

32. Unidades de Saúde Familiar - Contratualização 2008. Relatório Final. Porto: ARS Norte, IP - Departamento Contratualização; 2009. [documento na Internet]. [acedido 2010 jul. 10]. Disponível em: http://portal.arsnorte.min-saude.pt/portal/page/ portal/ARSNorte/Conte\%C3\%BAdos/Ficheiros/ Cuidados\%20Saude\%20Primarios/Relatorios/2008/ Relatorio_Contratualiza\%C3\%A7\%C3\%A3o_2008.pdf
33. Ferreira PL, Antunes P, Portugal S. O valor dos cuidados primários: Perspectiva dos utilizadores das USF. Lisboa: Ministério da Saúde; 2010.

34. Ferreira PL, Antunes P. Monitorização da satisfação dos utilizadores das USF. Centro de Estudos e Investigação em Saúde da Universidade de Coimbra (CEISUC) Maio de 2009. [documento na Internet]. [acedido 2010 jul. 10]. Disponível em: URL: http:// www.uc.pt/org/ceisuc/Documentos/USF_Util/ USF_Rel_

35. Ferreira PL, Antunes P. Monitorização da satisfação dos profissionais das USF. Coimbra: Centro de Estudos e Investigação em Saúde da Universidade de Coimbra (CEISUC); 2009. [documento na Internet]. [acedido 2010 jul. 10]. Disponível em: http:// www.uc.pt/org/ceisuc/Documentos/USF_Prof/ USF_Prof_Rel.pdf

36. Pisco, L. Main lines of action for reforming primary health care in Portugal. Primary Heath Care 2006; 15(2):63-66.

37. Pisco L. A reforma dos cuidados de saúde primários. Ordem dos Economistas. Cadernos de Economia 2007; XX(80)t: 60-66.

38. Pisco, L. Integrated care in Portugal. Revista de Innovación Sanitaria y Atención Integrada [periódico na Internet]. 2008 [acedido 2010 jul. 10]; 6(1):1. Disponível em: http://pub.bsalut.net/risai/vol1/iss1/6

39. Hurst J. Effective ways to realize policy reforms in health systems. Paris: Organization for Economic Co-operation and Development; 2010. [documento na Internet]. [acedido 2010 jul. 10]. Disponível em: http:// www.oecd.org/officialdocuments/displaydocumentpdf. (OECD Health Working papers $n^{\circ} 51$ ).

Artigo apresentado em 28/07/2010

Aprovado em 18/10/2010

Versão final apresentada em 09/12/2010 